\title{
CALCULATING THE FUNDAMENTAL GROUP OF AN ORBIT SPACE
}

\author{
M. A. ARMSTRONG
}

\begin{abstract}
Suppose $G$ acts effectively as a group of homeomorphisms of the connected, locally path connected, simply connected, locally compact metric space $X$. Let $\bar{G}$ denote the closure of $G$ in $\operatorname{Homeo}(X)$, and $N$ the smallest normal subgroup of $\bar{G}$ which contains the path component of the identity of $\bar{G}$ and all those elements of $\bar{G}$ which have fixed points. We show that $\pi_{1}(X / G)$ is isomorphic to $\bar{G} / N$ subject to a weak path lifting assumption for the projection $X \rightarrow X / \bar{G}$.
\end{abstract}

Given a topological space $X$ together with a group $G$ of homeomorphisms of $X$, what can we say about the fundamental group of the orbit space $X / G$ ? Results for simplicial and discontinuous groups have been given in [1] and [2]. The object of this note is to produce a theorem which can deal with both discontinuous and continuous actions.

We shall assume that $X$ is a connected, locally path connected, locally compact metric space. Let $G$ be a group of homeomorphisms of $X$ which acts effectively on $X$, so that we can think of $G$ as a subgroup of the group $\operatorname{Homeo}(X)$ of all homeomorphisms of $X$ endowed with the compact open topology.

Under very reasonable hypotheses (see conditions A, B, C below) the answer to our question is as follows. Let $\bar{G}$ denote the closure of $G$ in $\operatorname{Homeo}(X)$, and let $N$ be the smallest normal subgroup of $\bar{G}$ which contains the path component of the identity of $\bar{G}$ and all those elements of $\bar{G}$ which have fixed points. Then if $X$ is simply connected the fundamental group of $X / G$ is isomorphic to the quotient group $\bar{G} / N$.

Suppose $X$ fails to be simply connected but has a universal covering space $\tilde{X}$. Each homeomorphism $g: X \rightarrow X$ lifts to a homeomorphism of $\tilde{X}$, and any two lifts of the same $g$ differ by a covering transformation. Therefore we have an action of an extension of $\pi_{1}(X)$ by $G$ on $\tilde{X}$ whose orbit space is homeomorphic to $X / G$, and we can apply our result in this setting. Details of the group extension and of its action on $\tilde{X}$ can be found in [5] and [3].

Here are some examples to illustrate a variety of situations in which the result can be used.

EXAMPLE 1. Take $S^{2} \times \mathbf{R}$ for $X$ and $S^{1} \times \mathbf{Z}$ for $G$, the action being as follows. The circle acts on $S^{2}$ by rotation leaving the north and south poles fixed, and acts trivially on $\mathbf{R}$. The generator of $\mathbf{Z}$ reflects $S^{2}$ in the equator and translates $\mathbf{R}$ along

Received by the editors February 12, 1981.

1980 Mathematics Subject Classification. Primary 55Q05, 55599.

(C) 1982 American Mathematical Society $0002-9939 / 82 / 0000-0422 / \$ 02.25$ 
one unit. Then $G=\bar{G}, N=S^{1} \times\{0\}$ and the fundamental group of the orbit space (which is easily seen to be the Möbius strip) is $\mathbf{Z}$.

EXAMPLE 2. Consider the action of $G=\operatorname{PSL}(2, Z)$ on the upper half plane $U$ as a group of linear fractional transformations $z \mapsto(a z+b) /(c z+d)$. Then $G$ is a closed subgroup of $\operatorname{Homeo}(U)$, and is generated by the elements $z \mapsto-\frac{1}{z}$, $z \mapsto 1-\frac{1}{z}$ both of which have fixed points. Therefore the orbit space $U / G$ is simply connected. In fact the region

$$
\left\{z \in U|| z|\geqslant 1,| \operatorname{Re}(z) \mid \leqslant \frac{1}{2}\right\}
$$

is a fundamental region for the action of $G$, and looking at the way in which its sides are identified shows that $U / G$ is homeomorphic to a punctured sphere.

EXAMPLE 3. Consider the group of rationals $\mathbf{Q}$ acting on the real line $\mathbf{R}$ by addition. Taking the closure of $\mathbf{Q}$ in $\mathrm{Homeo}(\mathbf{R})$ gives a copy of $\mathbf{R}$ acting on itself by translation. Since $\mathbf{R}$ is path connected, $\mathbf{R} / \mathbf{Q}$ must be simply connected.

EXAMPLE 4. Let $G$ be a compact Lie group acting effectively on a simply connected space $X$. Assume that either $G$ is connected, or that $X^{G}$ (those points fixed by all elements of $G$ ) is nonempty. In both cases $G=\bar{G}=N$ and the orbit space $X / G$ must be simply connected.

EXAmple 5. Consider an irrational flow on the torus $T$. More precisely, let $\mathbf{R}$ act on $T \cong S^{1} \times S^{1}$ as follows: the real number $r$ sends

$$
\left(e^{2 \pi i x}, e^{2 \pi i y}\right) \text { to }\left(e^{2 \pi i(x+r)}, e^{2 \pi i(y+r \sqrt{2})}\right) \text {. }
$$

This action lifts to an action of $\pi_{1}(T) \times \mathbf{R}$ on $\mathbf{R}^{2}$ which has the same orbit space, namely $(m, n, r) \in \mathbf{Z} \times \mathbf{Z} \times \mathbf{R}$ sends $(x, y)$ to $(x+m+r, y+n+r \sqrt{2})$. One easily checks that the closure of this group of homeomorphisms of $\mathbf{R}^{2}$ is precisely the group of all translations of $\mathbf{R}^{2}$. Since this is a path connected group, the orbit space $T / \mathbf{R}$ is simply connected.

EXAMPLE 6. Let $F$ be a finitely generated free group and $X$ the graph of $F$ relative to a minimal set $S$ of generators. (That is to say $X$ has a vertex for each element of $F$, and an edge joins vertices $g$ and $h$ if and only if $h^{-1} g$ is an element of $S$ or the inverse of an element of $S$.) Note that $X$ is simply connected because $F$ is free. The action of $F$ on itself by left multiplication induces a free simplicial action of $F$ on $X$. If $G$ is any subgroup of $F$ we have an induced action of $G$ on $X$ and the orbit space $X / G$ is a one-dimensional simplicial complex. The fundamental group of this orbit space must therefore be free. But in terms of our result $G=\bar{G}$ and $N$ is the trivial subgroup, so this fundamental group is just $G$ and we recapture Nielsen's theorem that any subgroup of $F$ is free.

The conditions we shall need are listed below.
A. The projection $X \rightarrow X / \bar{G}$ has the path lifting property up to homotopy.
B. Given points $x, x^{\prime} \in X$ plus a neighborhood $V$ of $x$ in $X$, if $x^{\prime} \in \bar{G} x$ then $x^{\prime} \in G V$.
C. The group $\bar{G} / N$ acts discontinuously on $X / N$.

(The technical terms mentioned above are defined as follows. A map $f: X \rightarrow Y$ has the path lifting property up to homotopy if given a path $\alpha: I \rightarrow Y$, and a point $p \in f^{-1} \alpha(0)$, we can find a path $\beta: I \rightarrow X$ such that $\beta(0)=p, f \beta(1)=\alpha(1)$ and 
$f \beta \simeq \alpha \operatorname{rel}\{0,1\}$. A group $G$ acts discontinuously on a space $X$ if each point $x$ of $X$ has a neighborhood $V$ such that $g V \cap V$ is empty unless $g$ fixes $x$.)

Proposition 1. If condition $\mathrm{B}$ is satisfied then $X / G$ and $X / \bar{G}$ have the same homotopy type.

Proof. Let $\pi: X \rightarrow X / G$ and $\phi: X / G \rightarrow X / \bar{G}$ denote the natural projections. Define $\psi: X / \bar{G} \rightarrow X / G$ as follows; given $z \in X / \bar{G}$ choose a point $y \in X / G$ which satisfies $\phi(y)=z$ and set $\psi(z)=y$. Then $\phi \psi$ is the identity map of $X / \bar{G}$.

We need to show $\psi \phi$ homotopic to the identity map of $X / G$. To this end define $F: X / G \times I \rightarrow X / G$ by

$$
F(y, t)= \begin{cases}\psi \phi(y), & 0 \leqslant t<1, \\ y, & t=1 .\end{cases}
$$

If $F$ is continuous we are finished. Now given an open set $U$ of $X / G$ we have

$$
F^{-1}(U)=\left[\phi^{-1} \psi^{-1}(U) \times[0,1)\right] \cup[U \times\{1\}] .
$$

Clearly this is open in $X / G \times I$ if $U \subseteq \phi^{-1} \psi^{-1}(U)$, in other words if $\phi(U) \subseteq$ $\psi^{-1}(U)$.

Suppose $z \in \phi(U)$, say $\phi(y)=z$ where $y \in U$. We need to check that $\psi(z)$ lies in $U$. Let $\psi(z)=y^{\prime}$, then $\phi\left(y^{\prime}\right)=\phi(y)=z$. Choose $x, x^{\prime} \in X$ with images $y, y^{\prime}$ respectively in $X / G$. The set $V=\pi^{-1}(U)$ is an open neighborhood of $x$ in $X$, and $x^{\prime} \in \bar{G} x$ since $x$ and $x^{\prime}$ both map to $z$ in $X / \bar{G}$. By hypothesis we must have $x^{\prime} \in G V$. Therefore $\pi\left(x^{\prime}\right)$ lies in $\pi(G V)$, that is to say $\psi(z)$ lies in $U$ as required.

Corollary 2. If $G$ is a group of isometries of $X$, then $X / G$ and $X / \bar{G}$ have the same homotopy type.

Proof. Simply check condition B for a group of isometries. Suppose we have $x, x^{\prime} \in X$ such that $x^{\prime} \in \bar{G} x$, and let $V$ be a neighborhood of $x$ in $X$. Let $\varepsilon$ denote the distance from $x$ to $X-V$, and choose $g \in G$ such that $g(x)$ is within $\varepsilon$ of $x^{\prime}$. Then, since $g$ is an isometry, $x^{\prime} \in g V$ as required.

Condition $A$ is the only one which is hard to check, so we list several situations where it is satisfied.

Proposition 3. The projection $X \rightarrow X / \bar{G}$ has the path lifting property up to homotopy if any one of the following holds.

(a) $G$ acts simplicially on a triangulation of $X$.

(b) The action of $G$ on $X$ is discontinuous, and the stabiliser of any point is finite.

(c) $\bar{G}$ is a compact Lie group.

(d) $\bar{G}$ is a locally compact Lie group and acts properly on $X$.

(e) $X / \bar{G}$ is semilocally simply connected.

Proof. (a), (b), (c), (d) can be found in [1], [2], [3], [4], respectively, and in these cases one can actually lift the given path, rather than just a path which is homotopic to it. 
Case (e). We use an argument due to Smale [6], though our hypotheses are less restrictive. Let $\alpha: I \rightarrow X / \bar{G}$ be the given path, let $y_{0}=\alpha(0)$ and suppose $x_{0} \in$ $\pi^{-1}\left(y_{0}\right)$. For each point $y$ of $X / \bar{G}$ choose a neighborhood $U y$ such that loops in $U y$ are null homotopic in $X / \bar{G}$. We can do this since $X / \bar{G}$ is semilocally simply connected. Given $x \in \pi^{-1}(y)$ use the local path connectedness of $X$, and the continuity of $\pi$, to find a path connected neighborhood $P y(x)$ of $x$ such that $\pi(P y(x)) \subseteq U y$. If $x^{\prime} \in \pi^{-1}(y)$ also, set $P y\left(x^{\prime}\right)=g P y(x)$ where $g$ is an element of $\bar{G}$ which sends $x$ to $x^{\prime}$. Let $V y=\pi(P y(x))$ and note that $V y$ is a neighborhood of $y$ in $X / \bar{G}$ since $\pi$ is an open map.

Divide up $I$ into $N$ subintervals of equal length, taking $N$ sufficiently large so that $1 / N$ is a Lebesgue number for the open covering $\left\{\alpha^{-1}(V y) \mid y \in X / \bar{G}\right\}$ of $I$. Set $I_{1}=\{t \in I \mid 0 \leqslant t \leqslant 1 / N\}$ and note that $\alpha\left(I_{1}\right)$ must be contained in $V y$ for some point $y \in X / \bar{G}$. Therefore $y_{0} \in V y$ and $x_{0} \in P y(x)$ for some $x \in \pi^{-1}(y)$. Now $P y(x)$ is path connected, so we can join $x_{0}$ to some point of $P y(x) \cap$ $\pi^{-1}(\alpha(1 / N))$ by a path $\gamma: I \rightarrow P y(x)$. Define $\beta: I_{1} \rightarrow X$ by $\beta(t)=\gamma(N t)$. Then $\beta(0)=x_{0}, \pi(1 / N)=\alpha(1 / N)$, and $\pi \beta \simeq \alpha \mid I_{1} \operatorname{rel}\{0,1 / N\}$, since $\pi \beta$ and $\alpha \mid I_{1}$ have the same end points and both lie in $U y$. This defines our lift (up to homotopy) $\beta$ over $I_{1}$; the remaining subintervals are dealt with in a similar manner.

THEOREM 4. If $X$ is simply connected and if conditions $\mathrm{A}, \mathrm{B}$ and $\mathrm{C}$ are satisfied, then $\pi_{1}(X / G)$ is isomorphic to $\bar{G} / N$.

Proof. We shall show that $X / N$ is simply connected. Assume this is done and note that $\bar{G} / N$ acts freely on $X / N$, because $N$ contains all the elements of $\bar{G}$ which have fixed points, and that it acts discontinuously by hypothesis. Therefore the projection $X / N \rightarrow X / \bar{G}$ is a covering map and we deduce $\pi_{1}(X / \bar{G}) \simeq \bar{G} / N$. The theorem now follows from Proposition 1.

Choose a base point $p \in X$ and let $q=\pi(p)$, where $\pi$ now stands for the projection from $X$ to $X / N$. Define $\phi: N \rightarrow \pi_{1}(X / N, q)$ as follows. Given an element $g \in N$, join $p$ to $g(p)$ in $X$ by a path $\gamma$ and set

$$
\phi(g)=\langle\pi \circ \gamma\rangle \text {. }
$$

Notice that the choice of $\gamma$, amongst all paths joining $p$ to $g(p)$, is irrelevant since $X$ is simply connected. This function $\phi$ is a homomorphism, for given $g_{1}, g_{2} \in N$, and having chosen $\gamma_{1}$ joining $p$ to $g_{1}(p)$ and $\gamma_{2}$ joining $p$ to $g_{2}(p)$, use $\gamma_{1} \cdot g_{1}\left(\gamma_{2}\right)$ to join $p$ to $g_{1} g_{2}(p)$. Then

$$
\phi\left(g_{1} g_{2}\right)=\left\langle\pi \circ\left(\gamma_{1} g_{1}\left(\gamma_{2}\right)\right)\right\rangle=\left\langle\pi \circ \gamma_{1}\right\rangle\left\langle\pi \circ \gamma_{2}\right\rangle=\phi\left(g_{1}\right) \phi\left(g_{2}\right) .
$$

The kernel of $\phi$ is all of $N$. For if $g$ lies in the path component of the identity of $\bar{G}$, join $e$ to $g$ by a path $\left\{g_{t} \mid 0 \leqslant t \leqslant 1\right\}$ in $\bar{G}$. Then $\left\{g_{t}(p)\right\}$ joins $p$ to $g(p)$ in $X$ and projects to a single point in $X / N$. Therefore $g$ lies in the kernel of $\phi$. Now suppose $g$ is an element of $\bar{G}$ which fixes some point of $X$, say $g(x)=x$. Join $p$ to $x$ by a path $\gamma$, and use $\gamma(g \gamma)^{-1}$ to join $p$ to $g(p)$. This path projects to a null homotopic loop in $X / N$, so again $g$ lies in the kernel of $\phi$.

It only remains to show that $\phi$ is onto. Given an element of $\pi_{1}(X / N, q)$ represent it by a loop $\alpha$ based at $q$. Now $X \rightarrow X / \bar{G}$ has the path lifting property up 
to homotopy and, since $X / N \rightarrow X / \bar{G}$ is a covering map, so has $X \rightarrow X / N$. Therefore we can find a path $\beta$ in $X$ which begins at $p$ and which satisfies $\pi \beta(1)=q, \pi \beta \simeq \alpha \operatorname{rel}\{0,1\}$. Since $\beta(1) \in \pi^{-1}(q)$ there is an element $g \in N$ such that $g(p)=\beta(1)$, and by construction $\phi(g)=\langle\pi \circ \beta\rangle=\langle\alpha\rangle$. This completes the proof.

\section{REFERENCES}

1. M. A. Armstrong, On the fundamental group of an orbit space, Proc. Cambridge Philos. Soc. 61 (1965), 639-646.

2. The fundamental group of the orbit space of a discontimuous group, Proc. Cambridge Philos. Soc. 64 (1968), 299-301.

3. G. E. Bredon, Introduction to compact transformation groups, Academic Press, New York, 1972.

4. R. S. Palais, On the existence of slices for actions of non-compact Lie groups, Ann. of Math. (2) 73 (1961), 295-323.

5. F. Rhodes, On the fundamental group of a transformation group, Proc. London Math. Soc. (3) 16 (1966), 635-650.

6. S. Smale, A note on open maps, Proc. Amer. Math. Soc. 8 (1957), 391-393.

Department of Mathematics, University of DURham, Durham City, England 